\title{
Stepwise treatment for overactive bladder is prudent
}

\author{
Paul F. Austin, MD \\ Washington University School of Medicine, St. Louis Children's Hospital, St. Louis, MO, United States
}

Cite as: Can Urol Assoc J 2017;11 (1-2Suppl1):S80. http://dx.doi.org/10.5489/cuaj.4346

See related article on page S74.

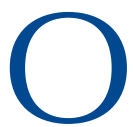

veractive bladder $(O A B)$ is a significant problem in children, as presented in this review by Ramsay and Bolduc. The prevalence of OAB is arguably underreported. The impact of $\mathrm{OAB}$ on the quality of life (QOL) cannot be ignored. Self-esteem, anxiety, and psychological distress are the primary indices of QOL impact and affect both the child and the caregiver or parents. Additionally, there is an economic impact beyond the direct cost of medical treatment for $O A B$ that includes the burden to change wet clothes, costs of diapers or pads, costs of excess laundry, and costs of time and travel involved with medical care that removes the child from school and the caregiver from work.

$\mathrm{OAB}$ is prevalent in both neurogenic- and non-neurogenic-type bladders and the treatments are similar, but there are some differences in the goals of medical therapy. The aim of medical therapy for OAB in both bladder types is to improve continence, but for neurogenic bladder, medical therapy is also aimed toward lowering detrusor storage pressures to an acceptably safe level in order to protect the upper urinary tract and prolong the time interval for clean intermittent catheterization (CIC).

It is important to note that there is a heterogeneous population with lower urinary tract (LUT) dysfunction and bladder and bowel dysfunction (BBD) that exhibit OAB. As highlighted, the impact of bowel dysfunction cannot be overlooked and is a significant comorbidity with LUT dysfunction. The key determination for OAB-directed treatment is the identification of the clinical and functional aspects of the bladder from the history, physical exam, and non-invasive urodynamics. For a small OAB with complete emptying, antimuscarinic and timed voiding/behavioural treatments/ education (standard urotherapy) are warranted and have excellent efficacy. For OAB with significant incomplete bladder emptying ${ }^{1}$ and history of urinary tract infections, anti- muscarinics are not as successful and standard urotherapy with possible biofeedback and/or neuromodulation (e.g., transcutaneous electrical nerve stimulation [TENS] unit) are warranted before giving an antimuscarinic that will potentially further impair bladder emptying. ${ }^{2}$ If $\mathrm{OAB}$ is still present after establishing complete emptying, then medical therapy will be more efficacious. This is analogous to converting a child with non-mono-symptomatic nocturnal enuresis to mono-symptomatic nocturnal enuresis (MSNE) before starting therapy aimed at MSNE.

As outlined in this review, stepwise medical treatment for $\mathrm{OAB}$ is prudent and aimed at either competitively antagonizing the binding of acetylcholine to the muscarinic receptors (antimuscarinics), agonizing or stimulating the beta-3 adrenergic receptors (mirabegron), or blocking the release of acetylcholine at the bladder neuromuscular junction (botulinum toxin). Other measures, which include forms of neuromodulation, should be considered thereafter. Finally, in patients with neurogenic bladder and an end-staged bladder with bladder wall fibrosis and poor detrusor compliance, medical therapy is unlikely to be effective and treatment involving surgical reconstruction is warranted.

Competing interests: Dr. Austin has been a clinical investigator for Allergan.

This paper has been peer-reviewed.

\section{References}

1. Chang SJ, Chiang IN, Hsieh CH, et al. Age- and gender-specific nomograms for single and dual post-void residual urine in healthy children. Neurourol Urodyn 2013;32:1014-8. https://doi.org/10.1002/ nau.22342

2. Thom $M$, Campigotto $M$, Coplen $D$, et al. Management of lower urinary tract (LUT) dysfunction: A stepwise approach, J Pediatr Urol 2012;8:20-4. https://doi.org/10.1016/i.jpurol.2011.01.004

Correspondence: Dr. Paul F. Austin, Washington University School of Medicine, St. Louis Children's Hospital, St. Louis, MO, United States; austinp@wudosis.wustl.edu 